\title{
Knowledge-based Lean Six Sigma Maintenance System for Sustainable Buildings
}

\author{
Abstract
}

Purpose- This paper develops a Knowledge-based (KB) System for Lean Six Sigma (LSS) Maintenance in environmentally Sustainable Buildings (Lean6-SBM).

Design/methodology/approach- The Lean6-SBM conceptual framework has been developed using the rule base approach of KB system and joint integration with Gauge Absence Prerequisites (GAP) technique. A comprehensive literature review is given for the main pillars of the framework with a typical output of GAP analysis.

Findings- Implementation of LSS in the sustainable building maintenance context requires a pre-assessment of the organisation's capabilities. A conceptual framework with a design structure is proposed to tackle this issue with the provision of an enhancing strategic and operational decision making hierarchy.

Research limitations/implications- Future research work might consider validating this framework in other type of industries.

Practical implications- Maintenance activities in environmentally sustainable buildings must take prodigious standards into consideration and, therefore, a robust quality assurance measure has to be integrated.

Originality/value - The significance of this research is to present a novel use of hybrid KB/GAP methodologies to develop a Lean6-SBM system. The originality and novelty of this approach will assist in identifying quality perspectives while implementing different maintenance strategies in the sustainable building context.

Keywords- Lean Six Sigma (LSS), Green Buildings, Sustainability, Building Maintenance, KB System (KBS), Gauge Absence Prerequisites (GAP)

Paper type- Research paper

\section{Introduction}

The main objective of any maintenance system is the achievement of maximal availability of a system with minimal cost (Mishra and Pathak, 2012). Their study proposes that this objective can be met by optimising the maintenance sub-objectives (e.g., enhancement of performance level, maximising operational efficiency, eliminating future defects, etc.), which are illustrated in Figure.1.

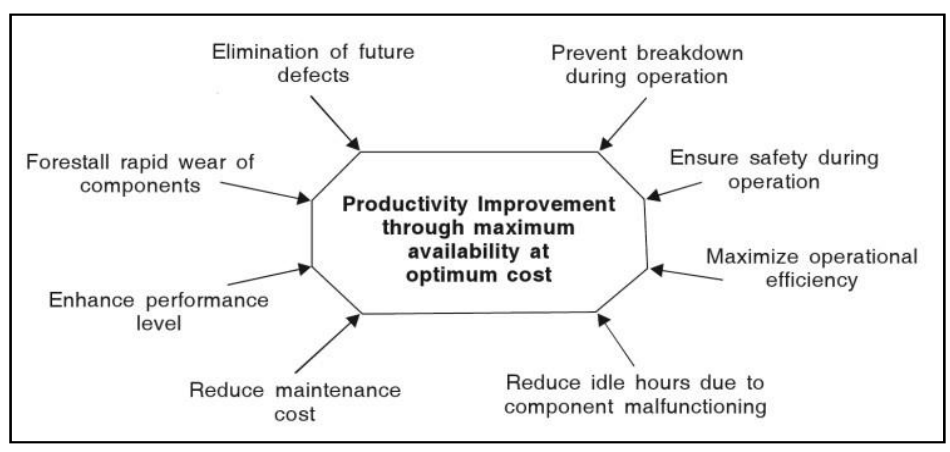

Figure. 1 Maintenance objectives, adopted from Mishra and Pathak (2012)

Maintenance oriented organisations spend substantial amounts of their annual budget in auditing and measuring their quality performance through hiring experts who, in many cases, are difficult tofind in very specialised areas(Macek and Dobiáš, 2014). According to Dhillon (2006), maintenance costs can represent between $60 \%$ to $75 \%$ of the life cycle cost for a large system. This creates a critical challenge for maintenance management to validate asset performance and allocate the required funds. High maintenance expenses occur because of 
insufficient reliability, which leads to frequent declines in service delivery (Salata et al., 2014).

Lind and Muyingo (2012) stated that public organisations have increased their focus on Building Maintenance (BM) because of huge investments in related infrastructure (e.g., hospitals and schools). However, different building components have different rates of deterioration, which in fact must have a scale of prioritisation in order to fulfil the main requirements to satisfy customer expectations (Olanrewaju and Abdul-Aziz, 2015).

The complexity of a sustainable BM environment and its related activities compel the top management level to determine a standardised universal performance audit that can be applied to all concerned departments. Currently, as part of performance auditing, quality management approaches vary from one organisation to another. This implies that much research has focused on measuring maintenance performance (Olanrewaju et al., 2011; Salata et al., 2014; Silva and Falorca, 2009; Wang et al., 2013) and continuous process improvement (Dukić et al., 2013; Suffian, 2013). However, these research initiatives have not assessed the performance of implementing integrated Lean and Six Sigma tools in sustainable BM. In practice, BM facilitators' approaches in measuring their maintenance quality management vary from regular inspections to advanced monitoring of Key Performance Indicators (KPI), using software applications such as Computerised Maintenance Management Systems (CMMS) and Enterprise Resource Planning (ERP).

According to Milana et al. (2014), unnecessary repairs or inspections definitely lead to increases in budgets. This indicates that maintenance processes are filled with non-valueadding steps that require continuous improvement. Therefore, there is a need to examine the integration of Lean with Six Sigma in such environments due to the fact that Six Sigma will address process control and customer focus with relevant tools, and Lean will accelerate the process by reducing the lead-time through elimination of waste (Albliwi et al., 2014).

A key aspect of current thinking is the use of green maintenance in Green Buildings. Green Building construction aims to minimise the total environmental impacts of this industry (Eichholtz et al., 2013). Therefore, it is possible to notice the concept of Green Building shifting towards the concept of sustainability, specifically environmentally sustainable buildings. This might justify the frequent use of the word green in the context of sustainable building. Approximately $51 \%$ of the engineers, architects, owners, contractors, and consultants who participated in a study, have anticipated that more than $60 \%$ of their work would lie in green technology construction by the year 2015 (Construction, 2013). Ding (2008) discussed the importance of comprehensive environmental building assessment methods, which assess building performance based on the triple bottom line of sustainability 
pillars (social, environmental, and economics aspects) and reflected the sustainability concept in the context of BM.

One of the main reasons behind weaknesses in maintenance management systems is the lack of experience which results in imprecise information obtained for decision making and hence, losing control of priorities. As an interesting example, when a certain item/asset needs to be declared for a major overhaul, although the manufacturer has put some default recommendations in the operation and maintenance manual (e.g., according to running hours), an expert site engineer may defer this action based on personal experience and practice that have been learned and understood. This might lead to a big saving (if he/she defers) or severe loss (if he/she does not defer) in machine performance, in addition to other financial impacts. This provides a reason for developing a Knowledge-based (KB) management system that can integrate Lean Six Sigma (LSS) as an advanced quality philosophy for sustainable building maintenance based on international best practice. The system will be embedded with Gauge Absence Prerequisites (GAP) technique to support benchmarking and decision making processes.

\section{Maintenance:}

Maintenance is defined by CIBSE Guide M (CIBSE-Guide-M, 2014)as "the combination of all technical and associated administrative actions intended to retain an item in, or restore it to, a state in which it can perform its required function". This leads to enhance the requirement of having maintenance performance measurements for buildings. Zawawi et al. (2011) insisted that performance in maintenance operations management had to be analysed and reviewed continuously in order to achieve high service quality. However, the traditional approach to achieving a high consistent performance leads to over-exhaustion of resources (Parida et al., 2015; Sahamir and Zakaria, 2014). These provide evidence of the need for a newer approach to overcome these problems.

LSS:

LSS is recognized as "a business strategy and methodology that increases process performance resulting in enhanced customer satisfaction and improved bottom line results"'(Snee, 2010). LSS is a quality philosophy that utilizes Lean management technique to speed up the process while applying Six Sigma (SS). This is performed by eliminating the non-value adding elements from the process. In fact, the whole process will be geared towards the minimum requirement of SS tools and techniques. Thus, Lean and SS are complementary to each other.

GAP Analysis:

GAP is a benchmarking tool that will be used in the Knowledge-based Lean6-SBM system. It will be used to assess the current situation of the company with the desired future situation (i.e. the benchmark) in order to estimate the performance gap between them. Khan and Hafiz 
(1999)and Milana et al. (2014) highlighted some generic objectives of a GAP analysis. From those objectives, two have motivated the use of GAP in BM environment. These include identifying weaknesses and strengths in current practices, and providing a quantitative basis for the existing system to be compared with the effective functioning of the desired process.

\section{Benefits of the $K B$ system:}

This research combines KB/GAP approaches in order to develop a conceptual framework of a hybrid Lean6-SBM system. Today, there is a dramatic increase in using KBSs in various disciplines. The reason is to reduce the high expenditures of hiring experts and to ease the knowledge transfer within an organisation, consequently improving productivity (Chang and Tsai, 2013; Grussing and Liu, 2013). The novel application of this approach to environmentally sustainable buildings will assist in identifying quality perspectives while implementing different maintenance strategies. It will go further by suggesting optimum and semi-optimum solutions based on experts' opinions and functional priorities. Thus, the research will deliver a framework for an effective decision support system that will assist top management, quality/maintenance managers and practitioners in the sustainable building maintenance sector to prioritise and monitor their performance and hence, increase productivity. In addition, the system will integrate LSS and a readiness evaluation framework to facilitate the implementation of this system.

\section{Literature Review}

The main elements of the proposed conceptual framework are Sustainable Building Maintenance, LSS, McKinsey's 7S framework, Expert System/ K B system, and Gauge Absence Prerequisite (GAP) and these will be discussed in this section in the light of recent and current research.

\subsection{Sustainable Building Maintenance}

Sustainable maintenance taxonomy and strategies are not independent of the conventional maintenance processes and practices in sustainable buildings. However, evidence in the literature highlights the need to be able to select different maintenance strategies according to the three sustainability pillars and their weightage criteria (Nezami and Yildirim, 2013). Alnaser (2008) outlined some advantages of sustainable building transformation: more energy-efficient performance, fewer emissions, less absenteeism, higher air quality, and longer lifecycle. According to Pulselli et al. (2007), building sustainability ensures resistance to physical degradation and hence maintains the main standard requirements in dynamic systems. On the other hand, Yahya and Ibrahim (2011) insist that health and safety play the most important role in forming sustainable building maintenance. According to Kaufman and Balsley (2009), maintenance staff must be trained to handle sustainable buildings. This will ensure the benefits of reduced long-term energy costs, efficient use of resources, and 
healthier employees. They further provided brief technical guidelines on how to maintain sustainable buildings.

Ajukumar and Gandhi (2013) have described how green maintenance has provided a means of making maintenance more environmentally friendly by getting rid of all associated wastes. They have emphasised that designers must consider the green aspects and design for ecofriendly maintenance. They also have classified the green maintenance requirements into three main categories: environmental compatibility, energy efficiency and human health and safety risks. These have been integrated with a prioritisation technique to evaluate their importance during maintenance operation. Chiang et al. (2015) have outlined an approach that can determine combinations of maintenance materials used while optimising life cycle cost (economic perspective), labour requirement (social perspective), and carbon emission (environmental perspective). However, one constraint at a time has to be chosen within acceptable levels while optimising the other two variables.

Building Owners and Managers Association (BOMA) 360 Certification was developed in 2009 with the aim of assessing any type of commercial building against reliability of operation and maintenance, including sustainability, risk assessment, safety issues, energy, and training. As a prerequisite, the organisation must have a standard operations manual and active preventive maintenance program in addition to a valid benchmark with a certified energy company (Penny, 2012). Despite the BOMA approach in building performance assessment, their evaluation sheet does not assign high weightage to maintenance. In addition, there is no reference to quality assessment in conjunction with maintenance.

In fact, some studies are beginning to deeply research the area of sustainable building maintenance. This can be supported by the clear evidence of a high rate in users' perceptions of sustainable building Baird (2015), the criticality of activating operation and maintenance manuals in low-carbon/sustainable buildings (Frank et al., 2015), and the guide to green maintenance and operation Kaufman and Balsley (2009)

\subsection{LSS}

LSS is as a methodology of business improvement that aims to maximise shareholder value by focusing on improving customer satisfaction, speed, quality, and cost(Franchetti, 2015; Laureani and Antony, 2011). In fact, it is a mix of tools and principles from Lean and Six Sigma that complement each other. The adoption and successful implementation of LSS has been reported at some international organisations (e.g., Motorola and GE). According to Zhang et al. (2012), LSS utilises Lean and Six Sigma tools and techniques to form a powerful remedial action that can eliminate the problems behind implementing each one of these approaches. Owing to the nature and complexity of this concept, which combines Lean and Six Sigma, there is a need to narrow their tools and techniques to suit the SBM environment. 
Some authors have demonstrated the importance of applying LSS in green maintenance and related construction projects. For example, Al-Aomar (2012) developed a Lean Six Sigma construction framework based on five KPIs; quality, cost, speed, value, and waste. The framework was tested in 28 construction companies and the findings revealed the amount of wastes and process variables that need to be tackled. Thomas et al. (2002) examined the impact of reducing the workflow variability in construction projects' performance. They found that those variables were available in all projects, even in the stages which were classified as very good in progressing. In maintenance practise, Wang et al. (2012) proposed a rigid traditional Define, Measure, Analyse and Control (DMAIC) framework that articulated the implementation of LSS in equipment maintenance process. They have identified some causes that consequently drove their approach. For example, they found that quality management was not standardised, inspection personnel made decisions by guesswork rather than data analysis, and most work was based on individual ability rather than teamwork. In fact, for the customised DMAIC stages, the factors which caused major LSS project failures needed to be addressed carefully.

Zhang et al. (2012) conducted a review of 116 papers on LSS and their findings revealed that most of the studies were focused on health process improvement, manufacturing, financial services, military equipment services, and other general services. However, there was no evidence of implementing LSS in building maintenance. In their findings from a survey conducted in 101 manufacturing and service companies, Laureani and Antony (2011) identified some critical success factors that could affect LSS implementation. The majority of respondents highlighted the importance of "leadership styles", "organisational culture", "management commitment", and "linking LSS to business strategy". Although the participant population was relatively small, the results were still valid in opening some gates for future research. This study has been enhanced by Albliwi et al. (2014), who designed a paper survey targeting previous studies (1995-2013) to investigate critical failure factors in Lean, Six Sigma, and LSS, revealed 34 factors that affected LSS implementation. These top factors were related to a lack of top management buy-in, lack of training, poor project selection, and lack of resources.

LSS has been internationally recognised as a powerful concept. However, there are still some arguments on how to proceed with a proper implementation strategy that the researcher believes will lead to catastrophic investment failure. It is obvious from previous studies that critical failure factors are just rotating around the three Cs of Oakland's soft elements described in Oakland (2014). These are: Communication, Commitment, and Culture; which, together, indicate the criticality of integrating the same in LSS. 
Despite the wide range of LSS successful implementations in manufacturing applications, there is no clear evidence in the literature of the integration of LSS in sustainable building maintenance through a KBS. Nevertheless, from a theoretical perspective, sustainable or green maintenance is highly related to eliminating waste and improving process variables. This enhances the need of integrating LSS in sustainable building context.

\subsection{Change Management Framework}

Different surveys indicate that more than $90 \%$ of projects conducted in Lean, Six Sigma, and LSS initiatives(Albliwi et al., 2014; Goh, 2012; Laureani and Antony, 2011) show both resistance to change and management commitment as key barriers to successful project implementation. Despite the built-in change management awareness process in the DMAIC model, there is a need for a comprehensive plan to assess and analyse the readiness to change in order to tackle such obstacles. These are mostly related to the entire process and human factors in BM environment like cross-functional support and training. Therefore, a McKinsey $7 \mathrm{~S}$ framework has been selected as a readiness test that will be integrated into the KB system. In fact, it proves the capability to deal with those factors.as stated by Hanafizadeh and Ravasan (2011).

\subsection{Expert System/ KB System}

An ES or a KBS is a decision making tool that automates using Artificial Intelligence (AI) methodologies. According to Khan et al. (2011), the terms ES and KBS have the same meaning; therefore, most scholars use them synonymously. When ESs were developed, they contained considerable knowledge regardless of whether this matched with the performance of human experts; therefore, they were called KBSs. Awad (1996) declared that for the ES, "the goal is to use specialised languages to design a computer-aided system based on an expert's thought process". The computer-aided system is the expert system shell that needs to be filled with a $\mathrm{KB}(\mathrm{KB})$. This $\mathrm{KB}$ contains rules, facts, and the acquired knowledge from human experts (Nawawi et al., 2008). In fact, these kinds of systems act as intelligent tools that in most cases should replace expertise in certain areas. KBSs are extensively used in many applications such as engineering, medicine, and banking. This gives an obvious indication of how powerful and reliable these systems are to ensure consistency in dealing with rapid decision making. One of the interesting application research studies was carried out by Khan et al. (2011) in which they developed a KB system facilitated by an analytical approach. The aim was to design a Flexible Manufacturing System (FMS) that helps in selecting the best possible arrangement of the equipment forming the FMS.

\subsection{Gauge Absence Prerequisite (GAP)}

The literature review provides evidence that the GAP analysis is a powerful benchmarking technique. For examples, it has been integrated with hybrid KB systems as a benchmarking 
tool in some areas, such as performance measurement systems (Khan et al., 2008), lean manufacturing (Nawawi et al., 2008), low volume automotive (Mohamed and Khan, 2012) and Maintenance Strategy and Operation (Milana et al., 2014). Therefore, this research will extend the use of GAP to the area of sustainable building maintenance in order to measure the differences between existing practices and the desired (Benchmark) ones. This will be articulated by using the GAP severity index adopted from Mohamed and Khan (2012) and tabulated as shown in Appendix A.

\section{Proposed Conceptual Framework of KB LSS Maintenance System for Sustainable Buildings (KB Lean6-SBM)}

Based on AlDairi et al. (2015), the KB Lean6-SBM conceptual model has been verified and validated in a conference paper. The feedback obtained is used to refine the model and consequently the related development steps as part of the verification and validation process. In addition, an extensive discussion has been carried out with the research supervisors, senior maintenance engineers, quality managers, and finally, LSS black belt and master black belt practitioners. The review with these experts has been extended to assure the critical selection of the KB Key Performance Indicators. This will enhance the project mission towards achieving the desired academic quality. The verification and validation process will be conducted once again, for the overall system after accomplishing the development of $\mathrm{KB}$ rules, through published and real industrial cases.

The validated KB Lean6-SBM model is converted into a suitable conceptual framework as shown in Figure. 2. The related KPIs will be utilised to generate the KB rules for different variables of LSS in SBM based on organisational hierarchy Levels of decision making. Finally, the rules will be stored in the KB database and facilitated by integration with the GAP analysis methodology to achieve optimal analysis and assessment outcomes of the decision making process. The design of the framework is set to assess the organisational capabilities from different perspectives, starting from a broad strategic Level and narrowing down to the most operational Level. As the study is targeting the implementation of LSS in a SBM context, it is necessary to study the critical success factors and critical failure factors involved in implementing LSS in a similar environment. From the extensive literature review and discussion with LSS practitioners, it has been found that the common factors that affect such implementation are the 3Cs driven by Oakland (2014); commitment, culture, and communication. These soft factors have to be addressed before making a decision to implement LSS. Thus, the findings indicate that for SBM to be successfully implemented, an initial reliable assessment of readiness in the form of McKinsey $7 \mathrm{~S}$ needs to be applied. The process is illustrated in Figure. 2: 


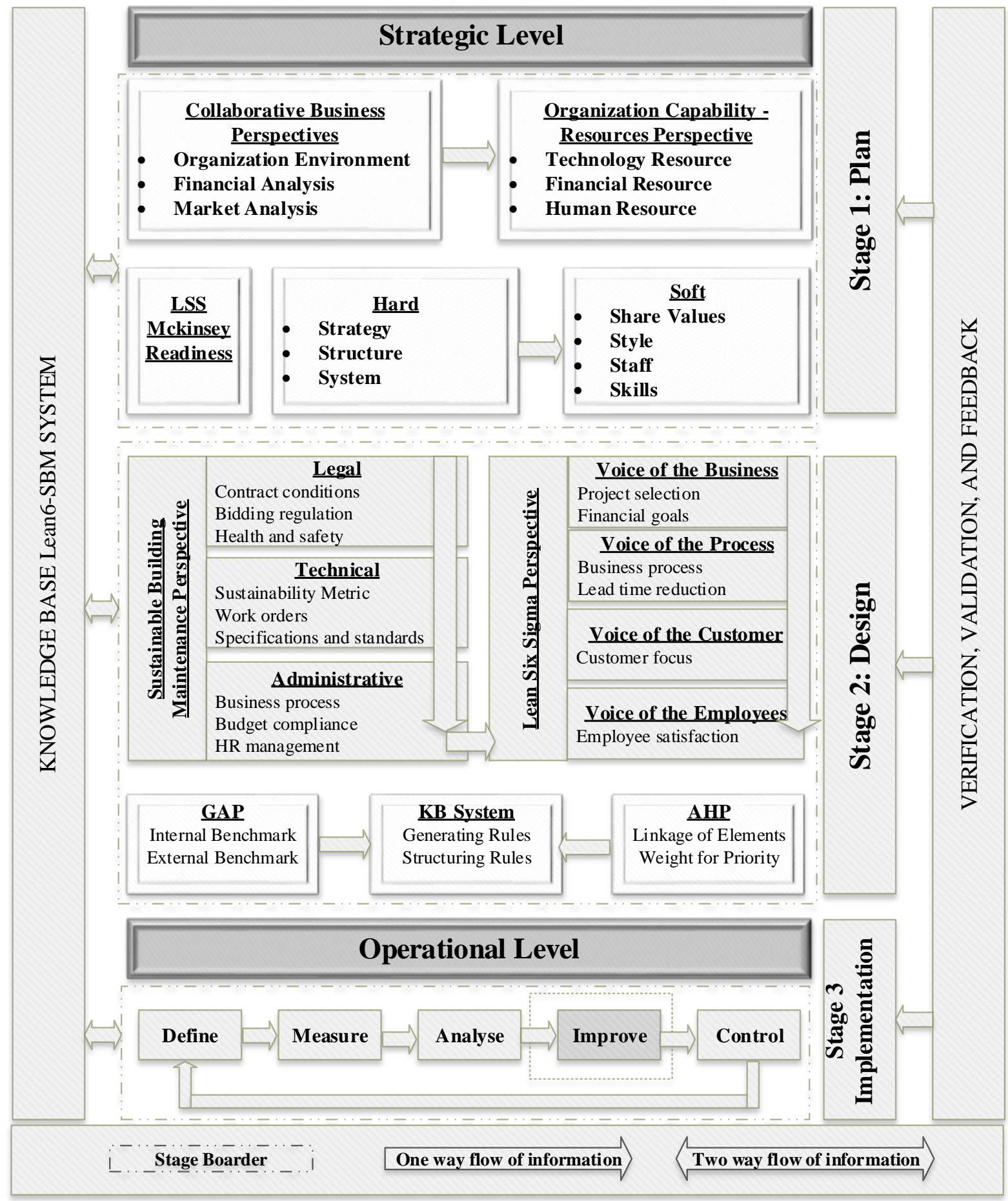

Figure.2 Lean6-SBM conceptual framework

This has to be integrated in the planning stage of the KB Lean6-SBM, after the identification of the organisation environment and its resources capabilities. To ensure the achievement of the main objectives of the study, it is very important for the organisation to truly commit its mission towards improving environmental performance. Therefore, it must consider the integration of environmental aspects in operation and decision making. 
The next stage within the framework is the designing stage. This stage has been designed to incubate the environmental sustainability aspects within a sustainable building maintenance taxonomy. The assessment starts with legal aspects in which contract condition is supported by the green/environmental national strategies, bidding regulations that will ultimately evaluate individuals and organisation ethics towards corporate social responsibilities (CSR), and finally health and safety aspects that will assess the overall practise of health, safety, and environment (HSE) regulations within the organisation.

With respect to technical aspects, there will be an evaluation of a number of sustainability measures that will determine how the maintenance teams perform their daily activities. These include, but are not limited to, the use of friendly and low emission tools and equipment, environmentally friendly products, green replacement process, green disposal process, measures of saving in energy consumption and effective use of transportation. The final aspects in SBM relate to administrative factors. In this aspect, the KB Lean6-SBM will assess the availability of documented business processes and how efficient is the commitment towards budget compliance. Furthermore, it will ensure the education and training process of employees based on adopting environmental-conscious practises that will help in minimising waste generation and energy consumption.

The integration of LSS in SBM is a complex process that requires clear attention and focus while planning to evolve such a KB. Thus for LSS, the critical path was to select appropriate Key Performance Indicators (KPIs) that led to having a comprehensive quality assessment process for a sustainable building maintenance context and was able to recommend optimum solutions to environmental issues. The designing stage is covered by multi-criteria decision making techniques used to facilitate Lean6-SBM.These are GAP analysis for benchmarking, and Analytic Hierarchy Process (AHP) for prioritisation. These will be embedded within the process of generating the system KB rules. According to Khan et al. (2008), AHP is widely applied as a powerful tool to weigh factors and for prioritising decisions in addition to confirming the integrity and correctness of those factors that are made by the user. AHP has been used for many quality and maintenance related applications (Ajukumar and Gandhi, 2013; Milana et al., 2014). However, it has been excluded from the scope of this paper as it will be part of the future work.

The last stage is the implementation which comes under the operational Level. In this stage, the DMAIC cycle is used to execute the selected projects after passing the initial assessments. According to Lin et al. (2013), DMAIC methodology can be explained as: Define business value and results along with customer needs using critical to quality (CTQ) 
or voice of customer (VOC) methods, Measure and validate data that help set priorities and criteria, Analyse to determine root causes and well understand the process and problem, Improve by developing solutions and refining goal statements, and finally Control and monitor the changes by developing a tracking process.

The implementation strategy in this research is built on extensive investigation of DMAIC key success and failure factors, which implies a DMAC (Define, Measure, Analyse, and Control) cycle as a screening type approach in which the project or issue must be tested against some prime conditions to distinguish whether it can be structured to meet the DMAIC criteria. It can be seen from the framework illustration in Figure. 2 that all of the stages are integrated with verification, validation, and feedback process. This will accelerate the system development process and enhance the capability of implementing the KB Lean6-SBM in real industries.

As a brief example of implementing LSS DMAIC cycle in a SBM context, a power distribution system (in a green building) has been selected as an asset type. Usually, the manufacturers recommend some standard preventive maintenance tasks to be carried out. This might include cleaning joints and bus-bars, ensuring the tightness of all connections, checking overloading and overheating of circuits...etc. However, one of the critical problems facing maintenance teams in power distribution systems is load balancing. This happens when currents flowing in hot wires (phases) are not equal, which leads to losing more power and therefore, a major financial impact that needs to be continuously measured, analysed and a control plan implemented accordingly. Various Six Sigma tools and techniques can be used to deal with such a case using the DMAIC cycle.

The identification of the problem is the first step in Define phase, followed by defining the expected benefits which need to be achieved. Then, a high level of the current power distribution maintenance process map (includes value-added, value-enabling, and non-valueadded steps) has to be drawn up and the possible primary and secondary metrics have to be documented along with a SIPOC (Suppliers Inputs Process Outputs Customers) diagram. The next stage is the Measure phase where the identified metrics need to be assessed using detailed process mapping, fishbone analysis, and FMEA (Failure Mode and Effect Analysis). The main task in this stage is to apply the Measurement System Analysis that detects repeatability and reproducibility errors within manpower and measurement devices. In the Analysis phase, the relationships between the variations and the variations with the output need to be driven using a statistical tool such as a Multi-Vari chart. This will, in turn, update the FMEA. The final step in this phase is to identify the root causes of the variations based on previous analysis by using a Pareto chart and cause and effect diagram. 
After defining, measuring, and analysing the existing load balancing process, the control chart can be utilised for monitoring where it can control both: Lower Specification Limit (LSL)/maximum load per phase and Upper Specification Limit (USL)/minimum load per phase using a long term data. Moreover, some green maintenance tasks within the system can be improved by applying Lean control tools and techniques. This will include the implementation of $5 \mathrm{~S}$ as initial step such as cleaning, applying colour coding and clear presentation of system circuit diagrams. As a result, the system faults' diagnosing and repairing processes will be much easier and the reduction of lead-time will be positively achieved. In addition to improving the financial goals, the reduction in power demand will proportionally affect the target of reducing the carbon footprint.

The system is designed structurally as illustrated in Figure. 3 to have effective decision making processes and hence a strong correlation must exist within the whole organisation departments. The framework has been delivered based on standard managerial organisation structures. Therefore, the proposed conceptual design structure has taken into account the hierarchy of decision making which will vary between strategic and operational levels. 


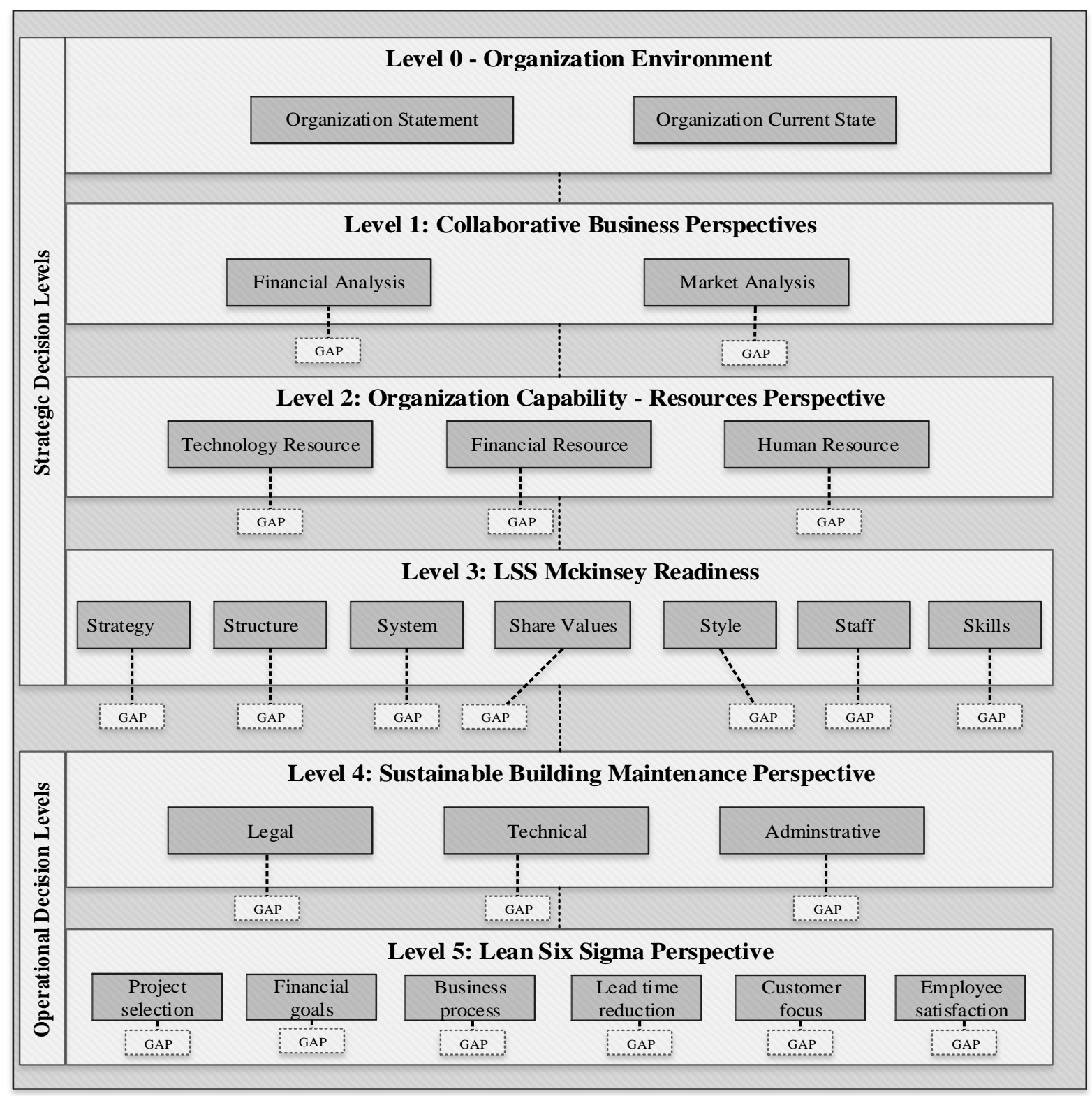

Figure.3 Lean6-SBM design structure

As seen in Figure. 3, the strategic issues fall under structure Levels 0 to 3, whereas the tactical and operational issues are more likely to be under Levels 4 and 5. Generally, the structural model represents the interrelation among all Levels (Level 0 to Level 5) and the areas in which to perform GAP analysis as a benchmarking technique.

\subsection{Level 0: Organisation Environment}

The organisational environment covers the basic strategic statements of the organisation. The organisation statement represents the expression of the organisation's initial identification. It specifies vision, mission, and business objectives that describe the bold guidelines of the business operation. Darbi (2012) concludes that vision and mission are strategic management tools that can affect employee attitudes and behaviours based on empirical studies. In addition to that, the organisational environment will capture the current 
situation of the BM organisation, including general information about the age of the organisation, number of employees, suppliers, customers, and number of competitors, which can be used to determine the size of the firm (Nawawi et al., 2008).

\subsection{Level 1: Collaborative Business Perspectives}

In order to achieve a comprehensive assessment of the SBM organisation, there must be an investigation into the organisation's business performance, which will be performed by inspecting the market and financial analysis. The financial analysis has critical importance in deriving the organisation's actual financial statement, affecting how well it will be able to deliver its KPIs. Based on the main objectives of SBM management, the key financial factors are Return on Asset (ROA), Return on Equity (ROE), and Return on Investment (ROI). These factors will be calculated to assess the organisation's health from a financial perspective (King and Lenox, 2001; Stefan and Paul, 2008). They are basically captured from the financial statements (i.e., balance sheet, income statement, and cash flow statement). According to Joo et al. (2011), the term ROA indicates how efficient the organisation is in using its assets to generate profits, while ROE reveals how much profit the organisation achieves with respect to shareholders' investment expressed as a percentage. Finally, ROI is used to evaluate the investment efficiency.

In parallel with the financial analysis, market analysis is categorised into market share and market place. The market share detects the percentage of business received from the customer base (Mohamed and Khan, 2012), whereas the market place highlights the area in which the maintenance service is applied, whether it be a restricted area, local, or global. This might influence the service lead-time that will be managed by LSS. Therefore, it is necessary to analyse the market performance and evaluate how well the organisation is attracting customers or clients through its services.

\subsection{Level 2: Organisation Capability - Resources Perspective}

Resource perspective assessment is critical in the SBM environment because it measures the capability of the organisation to cope with new changes. The three main pillars in this assessment are human resources, technology resources, and financial resources (Waldeck, 2014). With regard to human resources, the research will focus on three main dimensions: development, culture, and benefits. Human Resource Development (HRD) is "a process for developing and unleashing human expertise through organization development and personnel training and development for the purpose of improving performance" (Swanson and Holton, 2001). During the assessment process, the system will focus on three core elements within HRD: employee selection, training, and development planning. The importance of such attributes has been proven in terms of increasing productivity in construction companies (Tabassi et al., 2012), which is relatively close to the BM context.Tabassi et al. (2012) 
highlighted the importance of social systems (culture) and individual benefits in organisations and how these particularly affected knowledge transformation and general strategic objectives. Therefore, for the culture, the KB rules will seek the percentage of employee participation and involvement in decision making. In addition to that, the rules will benchmark the salary and other benefits within the overall benefits category.

The second resource pillar is technology, which is categorised into technology management, maintenance technology (Waldeck, 2014) and information and communication tools (Patterson et al., 2010). In technology management, the user (the user might be a quality/maintenance engineer, a supervisor, or any practitioner who will be able to answer the KB built-in questionnaire) will be asked about the organisation's maintenance objectives in addition to its development and process management. Next, the user will answer some questions related to maintenance technology from the angle of efficient tools and equipment in hand, latest defect measuring instruments and the availability and efficiency of the current asset management system. Finally, the user will be questioned on information and communication tools in order to determine the availability of an ICT master plan, network availability, and number of legacy systems.

The third resource pillar is financial resources, which address the budget allocation for employees, technology, and development (Mohd-Noor et al., 2011). With regard to employees, the organisation's capability will be assessed by checking the budget allocated for training and development, hiring, and benefits. On the other hand, focusing on technology involves checking the budget allocated for improving maintenance tools/equipment, maintaining the asset management system, and maintenance process improvement. Finally, from the development perspective, the assessment will trace the annual budget allocation to implement a quality management system during the previous three years.

\subsection{Level 3: LSS McKinsey Readiness}

The organisation's readiness will be tested prior to LSS implementation to highlight the degree of HR gap points. The McKinsey 7S framework technique will be embedded to enhance the LSS change management approach. Hanafizadeh and Ravasan (2011) indicated that McKinsey's 7S framework can be categorised into soft S's and hard S's. Soft S's are not easy to identify and comprise Staff, Skills, Style, and Shared Values, whereas hard S's are easy to identify and comprise Systems, Structure, and Strategy.

The LSS McKinsey Readiness Module is divided into hard S's and soft S's, with a systematic assessment process containing the necessary KPIs as recommended by Hanafizadeh and Ravasan (2011):

(1) Strategy KPIs contain vision and mission, goals and objectives (these must be well understood across the organisation) and a strategic maintenance plan. 
(2) Structure KPIs contain formalisation (the degree to which the procedures and rules are clearly documented and known by employees), centralisation (whether the organisation's decision making process is centralised or decentralised), and size and age of the organisation.

(3) Systems KPIs contain business processes, IT infrastructure, and ICT (Information and Communication Tools).

(4) Shared Values KPIs contain the project champion (who must continually lead the change management), cross-functional support (between departments), and shared beliefs (where all employees should be aware of the benefits of implementing LSS).

(5) Style KPIs contain commitment, communication, and culture.

(6) Staff KPIs contain HR management, training and education, and project team competencies.

(7) Skill KPIs contain management skills, LSS skills, and SBM skills.

\subsection{Level 4: Sustainable Building Maintenance Perspective}

According to Lind and Muyingo (2012), BM strategies can be divided into two types: corrective (e.g., planned and immediate) maintenance, which can be applied after detecting a fault, and preventive maintenance, which is applied before detecting the fault.Preventive maintenance is categorised into immediate, opportunistic, and planned, in which the maintenance is performed according to the manufacturer's recommendations (e.g., condition based, time based, planned opportunistic, and predictive). This research will focus on preventive maintenance and utilise the BM taxonomy driven by Motawa and Almarshad (2013), which comprises the following:

(1) Administrative Level, which contains the maintenance process and staff index

(2) Technical Level, which refers to the sustainability measures and technical work package

(3) Legal Level, which serves the contract conditions, bidding law, health, and safety

\subsection{Level 5: LSS Perspective}

The quality perspective will revolve around the basic philosophy requirements of LSS. According to Zhang et al. (2012), the LSS implementation is accomplished on a project basis in which each project must be completed in a time frame from 3 to 6 months. To ensure a successful implementation of this approach, specifically in the field of SBM, the LSS perspective is shown in Figure. 4 with the related KPIs. It contains project selection, and leadtime reduction, financial goals, business process, customer focus, and employee satisfaction (Aggogeri and Mazzola, 2008; e-Careers-Limited, 2013; Timans et al., 2012). 


\section{Examples of Generating KB Rules}

The following two examples will highlight in detail the process of creating KB rules followed by the typical outputs in the next section. They belong to the voices of the business and process from LSS perspectives. These sub-modules are project selection and lead-time reduction which will explore part of the importance of the scheme of applying LSS from environmental perspectives. Practically, both of these sub-modules are subjective and thus must be measured and converted into Critical to Quality (CTQ) in which the financial goals are easy to adjust according to the business objectives. For the KB system, Figure. 4 illustrates the system structure of the LSS perspective module with the related sub-modules.

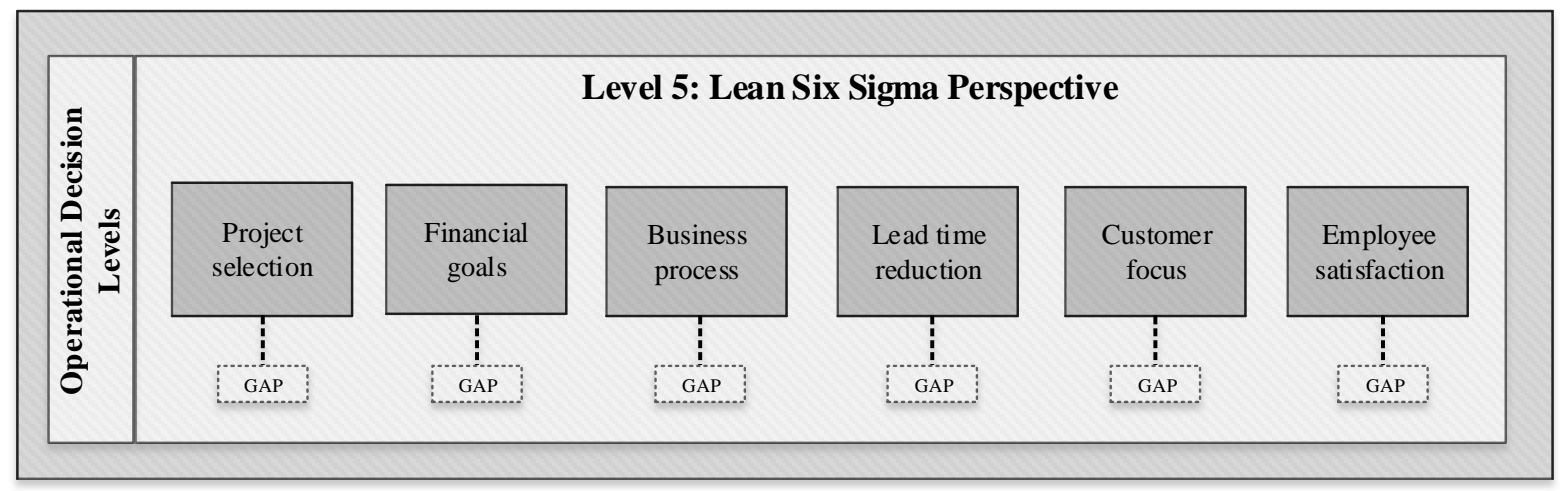

Figure. 4 The system structure of LSS perspective module

The user will be initially asked some questions (through the developed KB rules) that will determine whether the organisation has selected a valued project with clear financial goals. Then, the system will investigate the business process in conjunction with the SBM perspective module. This should explore the causes of change from the business process and lead-time perspectives. Subsequently, the user will be asked questions regarding customer focus and employee satisfaction. In fact, each KPI in this module is also linked to the information base as the data acquisition platform and benchmarked with the existing knowledge of best practises. Finally, the user feedback must be reviewed and verified at the end of the process. The results of the other LSS sub-modules (i.e.; financial goals, business process, customer focus, and employee satisfaction) will be shown as a summary in the typical output results.

\subsection{Project Selection Sub-module}

The main purpose of developing the project selection sub-module is to assess the validity of selecting the right project for implementing LSS in SBM context. By focusing on the project selection aspect, the important variables that will be evaluated are sustainability, clarity, data, and benefits. As described earlier, the main aim of this study is focused on implementing LSS in SBM context; therefore, the organisation has to be assessed as to whether it has taken 
the environmental aspects into account while selecting the project. This has to be enhanced by fulfilling the documented awareness policy according to international green/sustainability awarding certificates (e.g.; BREEAM, LEED). According to e-Careers-Limited (2013), clarity, data, and benefits are most essential for project selection. The problem must be documented and clear to all governance, the historical data which might be needed must be available and, finally, there must be a worthwhile benefit to the start-up the required project. The KB rule sets can be generated using the flowchart in Figure. 5.

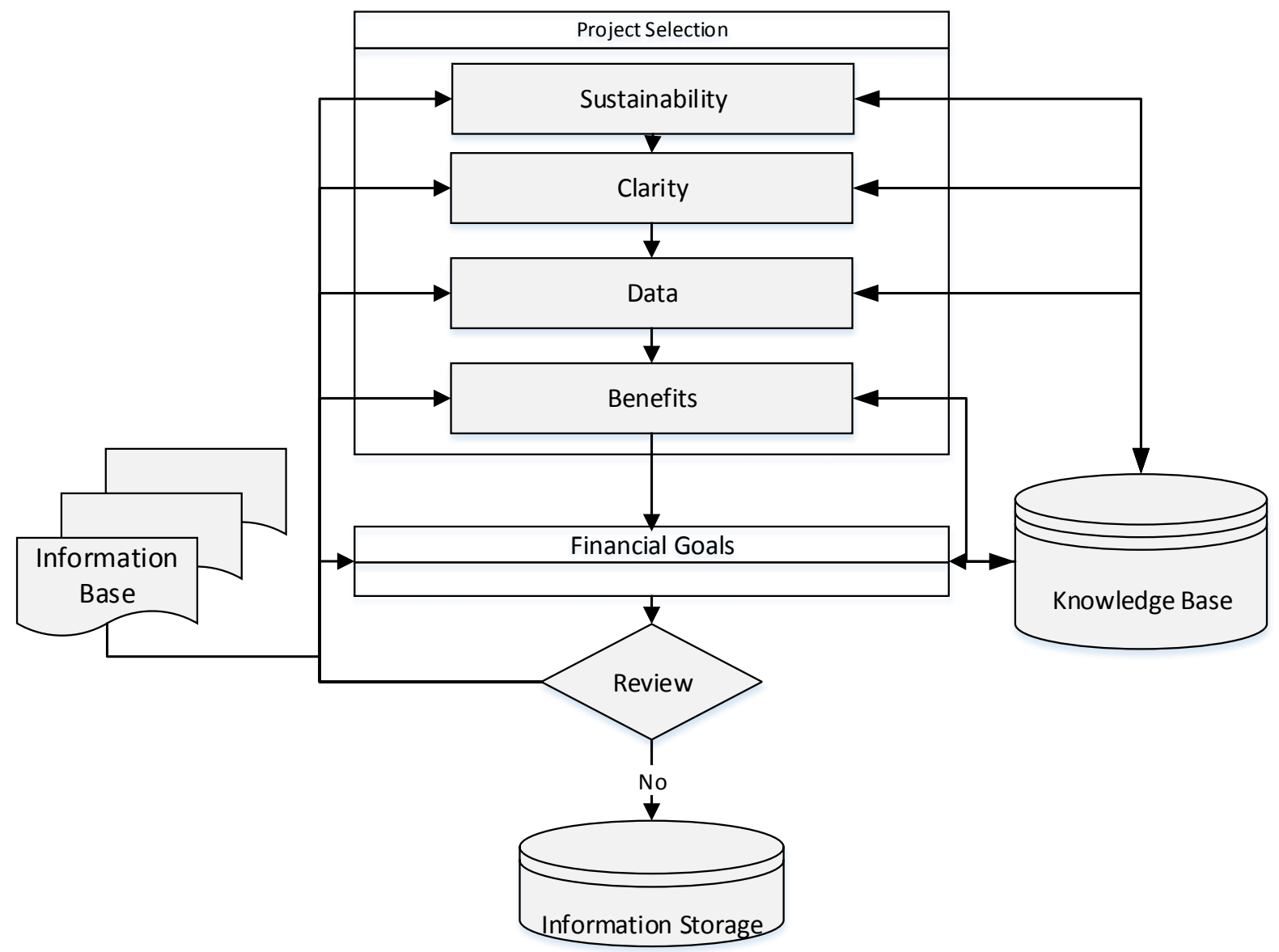

Figure. 5 The project selection sub-module

The following example demonstrates one of the KB rule sets in project selection sub-module:

IF the environmental policy of the Lean6-SBM organisation is documented and in line with the national sustainability strategies and CSR (Yes: GP; No: BP-PC-2)

AND the environmental targets are clearly defined and understood by organisation stakeholders (Yes: GP; No: BP-PC-2)

AND the Lean6-SBMorganisation has focused in environmental impacts of the process flow (Yes: GP; No: BP-PC-1)

AND the Lean6-SBMorganisationhas focused in social impacts of the process flow (Yes: GP; No: BPPC-1)

AND the Lean6-SBMorganisation has focused in economic impacts of the process flow (Yes: GP; No: BP-PC-1) 
AND the Lean6-SBMorganisation has taken into account the minimum consumption of natural resources (Yes: GP; No: BP-PC-1)

AND the historical data required to measure and forecast the energy consumption are consistently recorded (Yes: GP; No: BP-PC-1)

AND the historical data required to measure and forecast the waste production are consistently recorded (Yes: GP; No: BP-PC-1)

AND There is a conscious learning practise of sustainability benefits a cross employees and departments(Yes: GP; No: BP-PC-3)

THEN the Lean6-SBMorganisationhas a good mission and consideration towards sustainability measures

OR the organisation status is poor in respect to sustainability

The above KB set of rules demonstrates the importance of Lean6-SBM organisation in having the required awareness of sustainability. The organisation must be aware in optimising the three main pillars of sustainability: economic, environmental, and social impacts of the process flow. These are coded Good Points (GP) and Bad Points (BP). The importance here is to note and eliminate the Bad Points coded from 1-9 as Problem Categories to achieve Benchmarks (see Appendix A).

\subsection{Lead-time Reduction Sub-module}

In this sub-module, the main aim is to investigate the critical areas of improvements in respect to process. The mutual integration of Six Sigma with Lean will take place through evaluating the business process and lead-time reduction respectively. As this example is focusing on lead-time reduction, Figure. 6 illustrates the variables which need to be evaluated. These are correction, over-processing, transportation, motion, waiting, overproduction, and inventory (e-Careers-Limited, 2013) which are almost the same, as has been proven by Al-Aomar (2012). They stated that there were seven types of waste in the production and construction environment: delays, defects, excessive people movement, excessive transport, excessive inventory, over-production, and delivery of equipment and materials. Smith and Ball (2012) insisted that substantial improvements are needed to finite the use of natural resources, and reduce the generated emissions and wastes. These are outlined in Figure. 6. 


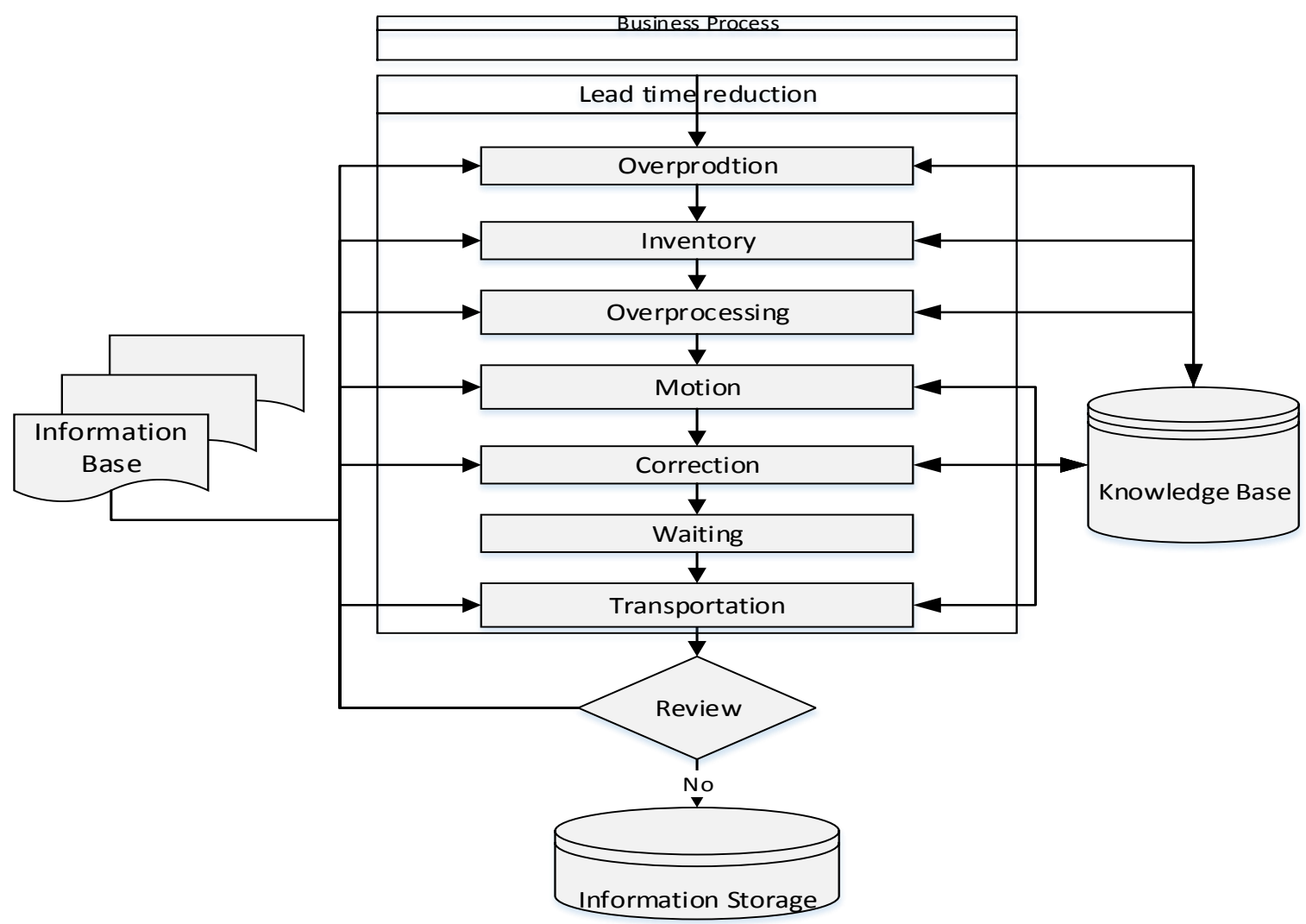

Figure. 6 The lead-time reduction sub-module

The following example demonstrates one of the $\mathrm{KB}$ rule sets in lead-time reduction submodule:

IF the Lean6-SBMorganisation has identified the best practice of waste elimination (benchmark) (Yes: GP; No: BP, PC-1)

AND the Lean6-SBMorganisation has assessed the aspects of $5 S$ (Sort, Set in order, Shine, Standardise, Sustain) (Yes: GP; No: BP, PC-1)

AND the Lean6-SBMorganisation has measurement records of lead-time overproduction process (Yes: GP; No: $B P$, PC-1)

AND the Lean6-SBMorganisation has records of number and percentage of out of quality (Yes: GP; No: BP, PC-1)

AND the Lean6-SBMorganisation has records of number and percentage of overproduction (Yes: GP; No: $\mathrm{BP}, \mathbf{P C}-\mathbf{1})$

AND the Lean6-SBMorganisation has records of number and percentage of energy used for the overproduction(Yes: GP; No: BP, PC-3)

AND the Lean6-SBMorganisation has records of average percentage of indoor air flow of the storage facility(Yes: GP; No: BP, PC-3)

AND the Lean6-SBMorganisationconsistently analyses the overproduction process (Yes: GP; No: BP, PC-1)

AND the Lean6-SBMorganisation consistently evaluates the lead-time in overproduction process (Yes: GP; No: BP, PC-1)

AND the Lean6-SBMorganisation consistently takes action to improve the lead-time in overproduction process (Yes: GP; No: BP, PC-1)

THEN the Lean6-SBMorganisation element of overproduction process is good and capable to achieve Lean6SBM alignment

OR the Lean6-SBMorganisation needs to reconsider its overproduction process to align the Lean6-SBM 
From the above rule sets, the Lean6-SBM organisation has to maintain some critical variations in order to be capable for over-production alignment. These are coded as PC-1 where the best practise of waste elimination has to be identified, the remedial $5 \mathrm{~S}$ concept is activated, and the over-production process lead-time is consistently analysed. Failure to perform these activities will result in a serious problem coded by PC-1.

\section{Typical Results and Analysis of Project Selection and Lead-time Reduction Sub-modules}

The KBLean6-SBM rules are initiated according to the sequentially designed questions asked to the organisation representative through a user interface linked to the KB application. This process is followed by demonstrating the output/results that will identify and pinpoint the aspects of weaknesses (Bad Points - BP) from the aspects of good practices (Good Points GP) according to the problem categories identified in Appendix-A. These problems are categorised with respect to severity, where PC- 1 is the most serious and indicates a very critical problem that need to be eliminated(Mohamed and Khan, 2012). The typical results of this study is shown in Table 1. For a simulation purpose, a total number of 544 questions (KB rules) have been asked. From these, 343 answers were implied as GPs and 201 number were detected as BPs. The BPs have been categorised as 73 PC-1, 34 PC-2, 25 PC-3, 12 PC-4, 11 PC-5, 6 PC-6, 6 PC-7, 8 PC-8, and 26 PC-9which need to be eliminated. These numbers of GPs and a breakdown of the numbers of BPs belong to LSS perspectives; project selection, financial goals, business process, lead-time reduction, customer focus, and employee satisfaction sub-modules. The problem categories for each sub-module of the Lean6-SBM is arranged in a prioritised manner.

In the project selection sub-module, the most critical area that needs to be focused on is sustainability since it has $3 \mathrm{PC}-1$. These are found to be related to not considering the environmental aspects while selecting a Lean6-SBM project. Therefore, it is a priority for the organisation to rectify these problems before taking action in the other 6 BP (1 PC-2, 2 PC-3, 1 PC-5, 1 PC-7, and 1 PC-9). The second critical area is Benefits which has the same number of PC-1 as Clarity but exceeds that of PC-2. Similarly, for the lead-time reduction submodule, it is very obvious that the main problem with Lean6-SBM organisation is in overprocessing which is indicated by $7 \mathrm{PC}-1$. This relates to adding more value to a product than the actual customer needs, such as painting an area that will never be seen or be exposed to corrosion. The second critical aspect is the inventory followed by the correction or the rework activities. Despite of the consequence relationship between overproduction and inventory, the rated problems seem to differ according to the pre-designed rule sets and the Lean6-SBM organisation representative answers. 
Table 1 Level 5 - LSS Perspective

\begin{tabular}{|c|c|c|c|c|c|c|c|c|c|c|c|c|c|}
\hline \multirow{2}{*}{ Sub-Module } & \multirow{2}{*}{ Dimension } & \multirow{2}{*}{$\begin{array}{c}\text { Number } \\
\text { of } \\
\text { Questions }\end{array}$} & \multirow{2}{*}{$\begin{array}{l}\text { Good } \\
\text { Points } \\
\text { (GP) } \\
\end{array}$} & \multirow{2}{*}{$\begin{array}{c}\text { Bad } \\
\text { Points } \\
\text { (BP) } \\
\end{array}$} & \multicolumn{9}{|c|}{ Bad Point Problem Category (PC) } \\
\hline & & & & & 1 & 2 & 3 & 4 & 5 & 6 & 7 & 8 & 9 \\
\hline \multirow[t]{5}{*}{$\begin{array}{l}\text { Project } \\
\text { Selection }\end{array}$} & Sustainability & 15 & 6 & 9 & 3 & 1 & 2 & 0 & 1 & 0 & 1 & 0 & 1 \\
\hline & Clarity & 17 & 9 & 8 & 2 & 2 & 1 & 0 & 2 & 0 & 0 & 1 & 0 \\
\hline & Data & 15 & 10 & 5 & 1 & 4 & 0 & 0 & 0 & 0 & 0 & 0 & 0 \\
\hline & Benefits & 18 & 7 & 11 & 2 & 3 & 0 & 1 & 1 & 0 & 1 & 1 & 2 \\
\hline & Sub Total & 65 & 32 & 33 & 8 & 10 & 3 & 1 & 4 & $\mathbf{0}$ & 2 & 2 & 3 \\
\hline \multirow{8}{*}{$\begin{array}{l}\text { Lead-time } \\
\text { reduction }\end{array}$} & Overproduction & 20 & 9 & 11 & 2 & 3 & 2 & 0 & 1 & 0 & 1 & 1 & 1 \\
\hline & Inventory & 18 & 10 & 8 & 5 & 0 & 1 & 0 & 1 & 0 & 0 & 0 & 1 \\
\hline & Over-processing & 16 & 7 & 9 & 7 & 1 & 1 & 0 & 0 & 0 & 0 & 0 & 0 \\
\hline & Motion & 19 & 11 & 8 & 2 & 5 & 0 & 0 & 0 & 0 & 0 & 0 & 1 \\
\hline & Correction & 20 & 10 & 10 & 4 & 0 & 0 & 0 & 0 & 0 & 0 & 0 & 6 \\
\hline & Waiting & 17 & 9 & 8 & 3 & 0 & 3 & 0 & 0 & 1 & 0 & 0 & 1 \\
\hline & Transportation & 15 & 10 & 5 & 0 & 0 & 5 & 0 & 0 & 0 & 0 & 0 & 0 \\
\hline & Sub Total & 125 & 66 & 59 & 23 & 9 & 12 & $\mathbf{0}$ & 2 & 1 & 1 & 1 & 10 \\
\hline $\begin{array}{l}\text { Financial } \\
\text { Goals }\end{array}$ & --- & 85 & 50 & 35 & 15 & 3 & 1 & 4 & 1 & 3 & 1 & 1 & 6 \\
\hline $\begin{array}{l}\text { Business } \\
\text { Process }\end{array}$ & --- & 90 & 60 & 30 & 13 & 5 & 4 & 2 & 1 & $\mathbf{0}$ & $\mathbf{0}$ & 2 & 3 \\
\hline $\begin{array}{l}\text { Customer } \\
\text { Focus }\end{array}$ & --- & 79 & 65 & 14 & 5 & 1 & 3 & $\mathbf{0}$ & 1 & $\mathbf{0}$ & $\mathbf{0}$ & 1 & 3 \\
\hline $\begin{array}{l}\text { Employee } \\
\text { Satisfaction }\end{array}$ & --- & 100 & 70 & 30 & 9 & 6 & 2 & 5 & 2 & 2 & 2 & 1 & 1 \\
\hline \multicolumn{2}{|c|}{ Grand Total } & 544 & 343 & 201 & 73 & 34 & 25 & 12 & 11 & 6 & 6 & 8 & 26 \\
\hline
\end{tabular}

Overall, it can be noticed that amongst these sub-modules, the project selection has the highest score $(51 \%)$ with regard to the number of BPs. This can be justified by the lack of awareness and documentation in regard to sustainability and the environmental benefits of the selected project. On the other hand, the business process and employee satisfaction reflect high values in respect to the percentage of the more critical points against the number of BPs.

\section{Conclusion}

This paper has presented a KB methodology for the Lean6-SBM system using a hybrid integration (KB, AHP and GAP) approach. The KB Lean6-SBM framework is designed to assess the organisation's capabilities through five Levels of different strategic and operational perspectives with a view to enhancing the performance of sustainable buildings. The strategic Level focuses on assessing the readiness of the organisation through the Mckinsey 7S framework while the operational Level evaluates the maintenance and LSS perspectives and their integration requirements. For this, $544 \mathrm{~KB}$ rules were developed. The 
process of generating $\mathrm{KB}$ rule sets is given with examples for LSS in environmental aspects (i.e.; project selection and lead-time reduction sub-modules). The results of the KB system for the LSS were presented and discussed. The project selection has scored the highest percentage of Bad Points and it has been found that the dimension of sustainability has the most critical part with 3PC-1, whereas for the lead-time reduction, the over-processing has scored 7PC-1. These results identify the key problems (GAPs) which need to be eliminated to achieve the Benchmarks. As remedial actions, the SBM organisation needs to focus more on fulfilling the environmental aspects while selecting a LSS project. Moreover, great attention has to be given to over-processing, with an action plan to implement a lean system into the daily green maintenance process. Thus, the $\mathrm{KB}$ system is a valid approach in assisting the decision making process in order to achieve Benchmarks in sustainability of Building Maintenance.

\section{Appendix A: Problem Categories and Description of GAP Technique}

\begin{tabular}{|c|c|c|}
\hline Category & Code & Description \\
\hline 1 & PC-1 & $\begin{array}{l}\text { This indicates a very serious problem, which should and can be resolved in the } \\
\text { short-term and the result of the problem is quite likely to provide a real short- } \\
\text { term benefits. }\end{array}$ \\
\hline 2 & PC-2 & $\begin{array}{l}\text { This indicates a serious problem, which involves pre-requisites to the system and } \\
\text { requires appropriate and logical improvement and implementation plan. }\end{array}$ \\
\hline 3 & PC-3 & $\begin{array}{l}\text { This indicates a major problem, which is likely to have pre-requisites to the } \\
\text { system and is better dealt with as part of an appropriate and logical } \\
\text { improvement and implementation plan. }\end{array}$ \\
\hline 4 & PC-4 & $\begin{array}{l}\text { This is quite a major problem, which is likely to have pre-requisites to the sub- } \\
\text { system and is better dealt with as part of an appropriate and logical } \\
\text { improvement and implementation plan. }\end{array}$ \\
\hline 5 & PC-5 & $\begin{array}{l}\text { This indicates a problem and can be dealt with now. If resolved, it is likely to } \\
\text { produce short-term benefits. }\end{array}$ \\
\hline 6 & PC-6 & $\begin{array}{l}\text { This indicates a minor problem and can be dealt with now. If resolved, it is likely } \\
\text { to produce short-term benefits. }\end{array}$ \\
\hline 7 & PC-7 & $\begin{array}{l}\text { This is not a serious problem. Although it could be dealt with now, it is unlikely to } \\
\text { produce short-term benefits. Therefore, it should only be dealt with if it is a pre- } \\
\text { requisite for other things. }\end{array}$ \\
\hline 8 & PC-8 & $\begin{array}{l}\text { This is not really a problem. However, it is important to consider certain } \\
\text { situations as future improvement. }\end{array}$ \\
\hline 9 & PC-9 & $\begin{array}{l}\text { This is not really a good or bad point itself. The questions associated with this } \\
\text { category are primarily asked to identify certain situations in the environment, } \\
\text { which upon subsequent probing by succeeding questions may well reveal } \\
\text { problems. }\end{array}$ \\
\hline
\end{tabular}

\section{References}

Aggogeri, F. and Mazzola, M. (2008) Combining six sigma with lean production to increase the performance level of a manufacturing system. American Society of Mechanical Engineers, pp. 425-434.

Ajukumar, V. N. and Gandhi, O. P. (2013) Evaluation of green maintenance initiatives in design and development of mechanical systems using an integrated approach. Journal of Cleaner Production, 51, $34-46$. 
Al-Aomar, R. (2012) A lean construction framework with Six Sigma rating. International Journal of Lean Six Sigma, 3 (4), 299-314.

Albliwi, S., Antony, J., Abdul Halim Lim, S. and van der Wiele, T. (2014) Critical failure factors of Lean Six Sigma: a systematic literature review. International Journal of Quality \& Reliability Management, 31 (9), 1012-1030.

Alnaser, N. W. (2008) Towards sustainable buildings in bahrain, kuwait and united arab emirates. Open Construction and Building Technology Journal, 2, 30-45.

Awad, E. M. (1996) Building Expert Systems: Principles, Procedures, and Applications. West Pub. Company.

Baird, G. (2015) Users' perceptions of sustainable buildings-Key findings of recent studies. Renewable Energy, 73, 77-83.

Chang, C.-Y. and Tsai, M.-D. (2013) Knowledge-based navigation system for building health diagnosis. Advanced Engineering Informatics, 27 (2), 246-260.

Chiang, Y. H., Li, J., Zhou, L., Wong, F. K. W. and Lam, P. T. I. (2015) The nexus among employment opportunities, life-cycle costs, and carbon emissions: a case study of sustainable building maintenance in Hong Kong. Journal of Cleaner Production, 109, 326-335.

CIBSE-Guide-M (2014) Maintenance Engineering and Management. United Kingdom: CIBSE.

Construction, M. H. (2013) World Green Building Trends: Business Benefits Driving New and Retrofit Market Opportunities in Over 60 Countries. Bedford Massachusetts: Smart Market Report.

Darbi, W. P. K. (2012) Of mission and vision statements and their potential impact on employee behaviour and attitudes: The case of a public but profit-oriented tertiary institution. International Journal of Business and Social Science, 3 (14), 95-109.

Dhillon, B. S. (2006) Maintainability, maintenance, and reliability for engineers. CRC Press.

Ding, G. K. C. (2008) Sustainable construction-The role of environmental assessment tools. Journal of environmental management, 86 (3), 451-464.

Dukić, D., Trivunić, M. and Starčev-Ćurčin, A. (2013) Computer-aided building maintenance with "BASE-FM" program. Automation in Construction, 30, 57-69.

e-Careers-Limited (2013) Lean Six Sigma Black Belt Manual XL v11. UK, An e-Careers Limited Publication.

Eichholtz, P., Kok, N. and Quigley, J. M. (2013) The economics of green building. Review of Economics and Statistics, 95 (1), 50-63.

Franchetti, M. J. (2015) Lean Six Sigma for engineers and managers. CRC Press.

Frank, O. L., Omer, S. A., Riffat, S. B. and Mempouo, B. (2015) The indispensability of good operation \& maintenance $(\mathrm{O} \& \mathrm{M})$ manuals in the operation and maintenance of low carbon buildings. Sustainable Cities and Society, 14, e1-e9.

Goh, T.-N. (2012) Some practical issues in the application of lean six sigma to service systems. Nang Yan Business Journal, 1 (1), 21-26.

Grussing, M. N. and Liu, L. Y. (2013) Knowledge-Based Optimization of Building Maintenance, Repair, and Renovation Activities to Improve Facility Life Cycle Investments. Journal of Performance of Constructed Facilities, 28 (3), 539-548.

Hanafizadeh, P. and Ravasan, A. Z. (2011) A McKinsey 7S model-based framework for ERP readiness assessment. International Journal of Enterprise Information Systems (IJEIS), 7 (4), 23-63.

J. AlDairi, M. K. Khan and Munive, J. E. (2015) A Conceptual Model for a Hybrid Knowledge-based Lean Six Sigma Maintenance System for Sustainable Buildings. In: Lecture Notes in Engineering and Computer Science: Proceedings of The World Congress on Engineering 2015, WCE 2015, 1-3 July. London, U.K, pp. 939-944.

Joo, S. J., Nixon, D. and Stoeberl, P. A. (2011) Benchmarking with data envelopment analysis: a return on asset perspective. Benchmarking: An International Journal, 18 (4), 529-542.

Kaufman, D. and Balsley, R. (2009) Aguide to Green Maintenance and Operations. Available from: http://www.stopwaste.org/docs/gbmg-dec-20-07ltr.pdf (Accessed 2 March 2015).

Khan, M., Wibisono and Dermawan (2008) A hybrid knowledge-based performance measurement system. Business Process Management Journal, 14 (2), 129-146.

Khan, M. K. and Hafiz (1999) Development of an expert system for implementation of ISO 9000 quality systems. Total Quality Management, 10 (1), 47-59.

Khan, M. K., Hussain, I. and Noor, S. (2011) A knowledge based methodology for planning and designing of a flexible manufacturing system (FMS). Int J Appl Manag Sci, 13 (1), 91-106.

King, A. A. and Lenox, M. J. (2001) Does it really pay to be green? An empirical study of firm environmental and financial performance: An empirical study of firm environmental and financial performance. Journal of Industrial Ecology, 5 (1), 105-116.

Laureani, A. and Antony, J. (2011) Standards for lean six sigma certification. International Journal of Productivity and Performance Management, 61 (1), 110-120. 
Lin, C., Chen, F. F., Chen, Y. M. and Kuriger, G. (2013) Continuous improvement of knowledge management systems using Six Sigma methodology. Robotics and Computer-Integrated Manufacturing, 29 (3), 95103.

Lind, H. and Muyingo, H. (2012) Building maintenance strategies: planning under uncertainty. Property Management, 30 (1), 14-28.

Macek, D. and Dobiáš, J. (2014) Buildings Renovation and Maintenance in the Public Sector. Procedia Engineering, 85, 368-376.

Milana, Khan and Munive (2014) A Framework of Knowledge Based System for Integrated Maintenance Strategy and Operation. Trans Tech Publ,Vol. 564, pp. 619-624.

Mishra, R. C. and Pathak, K. (2012) Maintenance Engineering and Management. PHI Learning.

Mohamed, N. M. Z. N. and Khan, M. K. (2012) The development of a hybrid knowledge-based system for the design of a Low Volume Automotive Manufacturing (LVAM) system. International Journal of Intelligent Systems Technologies and Applications, 11 (1), 17-35.

Mohd-Noor, N., Hamid, M. Y., Abdul-Ghani, A. A. and Haron, S. N. (2011) Building maintenance budget determination: an exploration study in the Malaysia government practice. Procedia Engineering, 20, 435-444.

Motawa, I. and Almarshad, A. (2013) A knowledge-based BIM system for building maintenance. Automation in Construction, 29, 173-182.

Nawawi, M., Khan, M. and Hussain, K. (2008) Knowledge-based collaborative lean manufacturing management (KBCLMM) system. Journal of KONBiN, 8 (1), 145-156.

Nezami, F. G. and Yildirim, M. B. (2013) A sustainability approach for selecting maintenance strategy. International Journal of Sustainable Engineering, 6 (4), 332-343.

Oakland, J. S. (2014) Total Quality Management and Operational Excellence: Text with Cases. Taylor \& Francis.

Olanrewaju, A. L., Khamidi, M. F. and Idrus, A. (2011) Structures of maintenance organisations in Malaysian universities. International Journal of Business Excellence, 4 (5), 573-594.

Parida, A., Kumar, U., Galar, D. and Stenström, C. (2015) Performance measurement and management for maintenance: a literature review. Journal of Quality in Maintenance Engineering, 21 (1), 2.

Patterson, M. K., Meakins, M., Nasont, D., Pusuluri, P., Tschudi, W., Bell, G. C., Schmidt, R., Schneebeli, K., Brey, T., McGraw, M., Vinson, W., Gloeckner, J. and Asme (2010) Energy-efficiency Through the Integration of Information and Communications Technology Management and Facilities Controls. (Ipack 2009: Proceedings of the Asme Interpack Conference 2009, Vol 2)

Penny, J. (2012) 10 Tips for Green Certification. Buildings, 106 (6), 36-40.

Pulselli, R. M., Simoncini, E., Pulselli, F. M. and Bastianoni, S. (2007) Emergy analysis of building manufacturing, maintenance and use: Em-building indices to evaluate housing sustainability. Energy and buildings, 39 (5), 620-628.

Sahamir, S. R. and Zakaria, R. (2014) Green Assessment Criteria for Public Hospital Building Development in Malaysia. Procedia Environmental Sciences, 20, 106-115.

Salata, F., de Lieto Vollaro, A., de Lieto Vollaro, R. and Davoli, M. (2014) Plant reliability in hospital facilities. Energy Procedia, 45, 1195-1204.

Silva, J. A. R. M. and Falorca, J. (2009) A model plan for buildings maintenance with application in the performance analysis of a composite facade cover. Construction and building materials, 23 (10), 32483257.

Smith, L. and Ball, P. (2012) Steps towards sustainable manufacturing through modelling material, energy and waste flows. International Journal of Production Economics, 140 (1), 227-238.

Snee, R. D. (2010) Lean Six Sigma-getting better all the time. International Journal of Lean Six Sigma, 1 (1), 929.

Stefan, A. and Paul, L. (2008) Does it pay to be green? A systematic overview. The Academy of Management Perspectives, 22 (4), 45-62.

Suffian, A. (2013) Some Common Maintenance Problems and Building Defects: Our Experiences. Procedia Engineering, 54, 101-108.

Swanson, R. A. and Holton, E. F. (2001) Foundations of human resource development. Berrett-Koehler Publishers.

Tabassi, A. A., Ramli, M. and Bakar, A. H. A. (2012) Effects of training and motivation practices on teamwork improvement and task efficiency: The case of construction firms. International Journal of Project Management, 30 (2), 213-224.

Thomas, H. R., Horman, M. J., de Souza, U. E. L. and Završki, I. (2002) Reducing Variability to Improve Performance as a Lean Construction Principle. Journal of Construction Engineering and Management, 128 (2), 144-154. 
Timans, W., Antony, J., Ahaus, K. and van Solingen, R. (2012) Implementation of Lean Six Sigma in small-and medium-sized manufacturing enterprises in the Netherlands. Journal of the Operational Research Society, 63 (3), 339-353.

Waldeck, N. E. (2014) Advanced manufacturing technologies and workforce development. Routledge.

Wang, L., Greenberg, S., Fiegel, J., Rubalcava, A., Earni, S., Pang, X., Yin, R., Woodworth, S. and HernandezMaldonado, J. (2013) Monitoring-based HVAC commissioning of an existing office building for energy efficiency. Applied Energy, 102, 1382-1390.

Wang, X., Wang, Y. and Xu, D. (2012) Lean six sigma implementation in equipment maintenance process. In: International Conference on Quality, Reliability, Risk, Maintenance, and Safety Engineering (ICQR2MSE). IEEE, pp. 1391-1395.

Yahya, M. R. and Ibrahim, M. (2011) Building maintenance policy issues in high rise commercial buildings.

Zawawi, E. M. A., Kamaruzzaman, S. N., Ithnin, Z. and Zulkarnain, S. H. (2011) A conceptual framework for describing CSF of building maintenance management. Procedia Engineering, 20, 110-117.

Zhang, Q., Irfan, M., Khattak, M. A. O., Zhu, X. and Hassan, M. (2012) Lean Six Sigma: a literature review. Interdisciplinary Journal of Contemporary research in business, 3 (10), 599-605. 